\title{
Graph congruences and what they connote
}

\author{
Izak Broere* and Lou Pretorius \\ Department of Mathematics and Applied Mathematics, University of Pretoria \\ e-mail: izak.broere@up.ac.za and loupretorius@gmail.com \\ Johannes Heidema ${ }^{\dagger}$ \\ Emeritus, Department of Mathematical Sciences, University of South Africa
}

\begin{abstract}
The algebraic notion of a "congruence" seems to be foreign to contemporary graph theory. We propound that it need not be so by developing a theory of congruences of graphs: a congruence on a graph $G=(V, E)$ being a pair $(\sim, \widehat{E})$ of which $\sim$ is an equivalence relation on $V$ and $\widehat{E}$ is a set of unordered pairs of vertices of $G$ with a special relationship to $\sim$ and $E$. Kernels and quotient structures are used in this theory to develop homomorphism and isomorphism theorems which remind one of similar results in an algebraic context. We show that this theory can be applied to deliver structural decompositions of graphs into "factor" graphs having very special properties, such as the result that each graph, except one, is a subdirect product of graphs with universal vertices. In a final section, we discuss corresponding concepts and briefly describe a corresponding theory for graphs which have a loop at every vertex and which we call loopy graphs. They are in a sense more "algebraic" than simple graphs, with their meet-semilattices of all congruences becoming complete algebraic lattices.
\end{abstract}

Keywords: graph, congruence, homomorphism, quotient graph, isomorphism theorem, subdirect product, loopy graph, universal vertex, complete algebraic lattice

2010 Mathematics Subject Classification: 05C25, 05C76

\section{Preliminaries}

For those notions on graphs in general not defined here, we refer the reader to [2]. Except when explicitly stated otherwise (as in Section 5), all graphs considered are simple, undirected and unlabelled, and have non-empty vertex sets, with no upper bound on their cardinalities.

A graph $G$ with vertex set $V$ and edge set $E$ will typically be denoted by $G=(V, E)$; when we are dealing with different graphs, we may use the notation $V(G)$ or $V_{G}$ for $V$ and, similarly, $E(G)$ or $E_{G}$ for $E$. An independent set (of vertices) in a graph induces an edgeless subgraph of the graph. A (graph) homomorphism is an edge preserving mapping from the vertex set of a graph into the vertex set of a graph. When two graphs are isomorphic, one will be called a clone of the other.

If $x$ and $y$ are elements of some set, we shall denote an ordered pair formed by them by $(x, y)$ and the unordered pair $\{x, y\}$ formed by them by $x y$.

Congruences: Consider any graph $G=(V, E)$. A congruence on $G$ is a pair $\theta=(\sim, \widehat{E})$ such that (i) $\sim$ is an equivalence relation on $V$ (hence $i d_{V} \subseteq \sim$ );

(ii) $\widehat{E}$ is a set of unordered pairs of different elements from $V$ with $E \subseteq \widehat{E}$;

(iii) when $x \sim y$ then $x y \notin \widehat{E}$ (i.e., $\sim$-equivalence classes are independent sets of vertices with respect to $\widehat{E})$; and

(iv) when $x, y, x^{\prime}, y^{\prime} \in V, x \sim x^{\prime}, y \sim y^{\prime}$, and $x y \in \widehat{E}$, then $x^{\prime} y^{\prime} \in \widehat{E}$.

\footnotetext{
${ }^{*}$ Corresponding author. Supported in part by the National Research Foundation of South Africa (Grant Number 90841).

${ }^{\dagger}$ Johannes Heidema passed away unexpectedly on 11 February 2017.
} 
We shall refer to condition (iv) above as the substitution property of $\widehat{E}$ with respect to $\sim$. After Corollary 2 in Section 3 there are some comments on the role and necessity of $\widehat{E}$ in congruences.

When $x \in V$ we define (as is usual) $[x]_{\sim}:=\{y \in V \mid x \sim y\}$ and if $\sim$ is understood from the context, we write simply $[x]$ for $[x]_{\sim}$. The congruence $\iota_{G}:=\left(i d_{V}, E\right)$ on $G$ is obviously the smallest congruence on $G$, i.e., the $\subseteq$-smallest in both components, and is called the identity congruence on $G$.

Examples: A congruence can easily be constructed on any given graph $G=(V, E)$ by taking, for any two vertices $x$ and $y$ of $G, x \sim y$ to mean $x=y$ and $x y \in \widehat{E}$ to mean that there is a (finite) path from $x$ to $y$ in $G$. Should one think of $(V, \widehat{E})$ as a graph, then it is the graph obtained from $G=(V, E)$ by replacing each connected component of $G$ by a complete graph on the vertex set of that component. More generally: the construction on a graph $G=(V, E)$ of leaving $V$ intact while extending $E$ to some edge set $\widehat{E}$, yielding the new graph $(V, \widehat{E})$, corresponds to the congruence $(=, \widehat{E})$ on $G$. Just such constructions are employed in the proof of Theorem 6 in Section 4.

For another example, again given $G=(V, E)$, one could choose a non-empty independent set of vertices, $W \subseteq V$, and define a congruence $\theta=(\sim, \widehat{E})$ on $G$ by stipulating

$$
\begin{aligned}
& \sim:=i d_{V \backslash W} \cup(W \times W) \text { and } \\
& \widehat{E}:=\left\{x y \mid[x, y \in V \backslash W \text { and } x y \in E] \text { or }\left[x \in V \backslash W, y \in W \text { and }\left(\exists y^{\prime}\right)\left(y^{\prime} \in W \text { and } x y^{\prime} \in E\right)\right]\right\} .
\end{aligned}
$$

Then one should think of constructing a new graph, to be denoted next by $G / \theta$, with the vertex set and edge set

$$
\begin{aligned}
& V(G / \theta):=(V \backslash W) \cup\{W\}=V / \sim \text { and } \\
& E(G / \theta):=\{x y \mid x y \in E ; x, y \in V \backslash W\} \cup\{x W \mid x \in V \backslash W \text { and }(\exists y)(y \in W \text { and } x y \in E)\}
\end{aligned}
$$

respectively. (Please allow here a small innocuous ambiguity, namely identifying $\{x\}=[x]_{\sim}$ and $x$ for $x \in V \backslash W$.) We note that the graph $G / \theta$ in this example is obtained by identifying the vertices of the pre-given independent set $W$.

The latter example serves as a precursor for the next definition.

Quotients: Given any congruence $\theta=(\sim, \widehat{E})$ on a graph $G=(V, E)$, we define a new graph, denoted by $G / \theta$ and called (the quotient of) $G$ modulo $\theta$, as follows:

$$
\begin{aligned}
G / \theta & :=(V(G / \theta), E(G / \theta)) \\
& :=(V / \sim,\{[x][y] \mid x y \in \widehat{E}\}) .
\end{aligned}
$$

We note that the surjective mapping $x \mapsto[x]$ from $V(G)$ onto $V(G / \theta)$ establishes the natural or canonical homomorphism $G \longrightarrow G / \theta$. Note also that $G / \iota_{G} \cong G$.

Direct products and subdirect products: The direct product $\Pi_{i \in I} G_{i}$ of a set $\left\{G_{i} \mid i \in I\right\}$ of graphs is obtained by taking as vertex set $V\left(\Pi_{i \in I} G_{i}\right)=\prod_{i \in I} V\left(G_{i}\right)$, the Cartesian product of the vertex sets of the graphs $G_{i}$, while two vertices $f$ and $g$, which are both functions from $I$ to $\bigcup_{i \in I} V\left(G_{i}\right)$ and which satisfy $f(i) \in V\left(G_{i}\right)$ and $g(i) \in V\left(G_{i}\right)$ for all $i \in I$, are adjacent in $\Pi_{i \in I} G_{i}$ if and only if $f(i) g(i) \in E\left(G_{i}\right)$ for all $i \in I$. For such a direct product $\Pi_{i \in I} G_{i}$, the $i$ th projection from $\Pi_{i \in I} G_{i}$ to $G_{i}$ is the mapping $\pi_{i}: f \mapsto f(i)$; note that these projections are all (graph) homomorphisms. A subdirect product of a set of graphs $\left\{G_{i} \mid i \in I\right\}$ is an induced subgraph of the direct product $\Pi_{i \in I} G_{i}$ of these graphs for which the restriction (to that subgraph) of every $i$ th projection is surjective onto $G_{i}$.

Preview: Using these concepts, we develop an "algebraic" theory of graphs which reminds one of the theories in modern algebra and universal algebra, explicated for example in [3] and [5]. By defining some of the most fundamental concepts for an algebraic approach to graph theory and proving some of the basic concomitant theorems, we hope to evoke further work in this vein.

In Section 2 we investigate the posets of all congruences on a graph and of all those congruences larger than a fixed congruence on the graph.

In Section 3 we formulate and prove the First Isomorphism Theorem for Graphs. This is followed by an explanation of why the notion of a congruence on a graph, a relational structure, needs to be 
more involved than the corresponding notion on an algebraic structure, with operations. The Second Isomorphism Theorem for Graphs and the Third Isomorphism Theorem for Graphs are then derived as applications of the First Isomorphism Theorem for Graphs.

In Section 4 we employ congruences and quotient graphs to show a general relationship between the infimum of a set of congruences on a graph and a subdirect product of the quotient graphs modulo those congruences. Here it can be demonstrated that every graph, except the two-vertex graph with no edge, is a special subdirect product of graphs by showing that one can ask that each of the graphs used has a universal vertex.

In a final section we briefly consider a corresponding theory by deriving similar results for graphs which have a loop at every vertex, here called loopy graphs. It is an easy exercise to develop analogously a theory for directed graphs. Taking some care, multiple edges and labels on edges and on vertices could be accommodated.

We note that the proper generalization of the notion of a "congruence" from algebra to structures with operations, partial operations, and relations - of which we employ a special case - originates in [4].

\section{The poset of congruences on a graph}

We now consider the natural partial ordering on the set of all congruences on $G$. Let us denote the set of all congruences on a graph $G=(V, E)$ by $\mathcal{C}(G)$. We note that the relation $\sqsubseteq$, defined on $\mathcal{C}(G)$ by

$$
(\sim, \widehat{E}) \sqsubseteq\left(\sim^{\prime}, \widehat{E^{\prime}}\right) \text { if and only if } \sim \subseteq \sim^{\prime} \text { and } \widehat{E} \subseteq \widehat{E^{\prime}}
$$

for all pairs of congruences from $\mathcal{C}(G)$, is a partial order relation on $\mathcal{C}(G)$. More than that: If $\theta_{i}=\left(\sim_{i}, \widehat{E_{i}}\right)$, $i \in I$, are given congruences on $G$, one can easily see that the pair $(\sim, \widehat{E})$ defined by $\sim:=\bigcap\left\{\sim_{i} \mid i \in I\right\}$ and $\widehat{E}:=\bigcap\left\{\widehat{E_{i}} \mid i \in I\right\}$ is a congruence on $G$.

Hence we can define

$$
\bigwedge\left\{\theta_{i} \mid i \in I\right\}:=\left(\bigcap\left\{\sim_{i} \mid i \in I\right\}, \bigcap\left\{\widehat{E_{i}} \mid i \in I\right\}\right)
$$

and note that this is the greatest lower bound of the given congruences in $(\mathcal{C}(G)$; $\sqsubseteq)$. This ensures that $(\mathcal{C}(G) ; \sqsubseteq)$ is a (complete) meet-semilattice, since arbitrary meets exist in it. With respect to joins, one can only remark that every set of congruences in $\mathcal{C}(G)$ which is bounded above in $(\mathcal{C}(G)$; $)$ has a least upper bound in $(\mathcal{C}(G) ; \sqsubseteq)$ - the meet of all such upper bounds.

Should $\mathcal{C}^{\prime} \subseteq \mathcal{C}(G), \mathcal{C}^{\prime}=\left\{\theta_{i} \mid i \in I\right\}$ with $\theta_{i}=\left(\sim_{i}, \widehat{E_{i}}\right)$, be a $\sqsubseteq$-chain in $\mathcal{C}(G)$ - i.e., for all $i, j \in I$, $\theta_{i} \sqsubseteq \theta_{j}$ or $\theta_{j} \sqsubseteq \theta_{i}$ - then $\left(\bigcup_{i \in I} \sim_{i}, \bigcup_{i \in I} \widehat{E_{i}}\right)$ is an upper bound for $\mathcal{C}^{\prime}$ in $(\mathcal{C}(G)$; $)$. We verify explicitly that the purported upper bound satisfies condition (iii) on congruences. Suppose that $(x, y) \in \bigcup_{i \in I} \sim_{i}$ and in particular that $x \sim_{j} y$. Then (by (iii) for $\left.\theta_{j}\right) x y \notin \widehat{E_{j}}$. Consider any $i \in I, i \neq j$. If $\theta_{i} \sqsubseteq \theta_{j}$, then $\widehat{E_{i}} \subseteq \widehat{E_{j}}$, with $x y \notin \widehat{E_{i}}$. If $\theta_{j} \sqsubseteq \theta_{i}$, then $\sim_{j} \subseteq \sim_{i}$; hence $x \sim_{i} y$ and $x y \notin \widehat{E_{i}}$. So $x y \notin \bigcup_{i \in I} \widehat{E_{i}}$ and (iii) holds; and so do (i), (ii), and (iv). Since this upper bound is made up of unions, every chain in $\mathcal{C}(G)$ clearly has an upper bound, in fact, a least upper bound in $\mathcal{C}(G)$. Therefore, by the grace of Zorn's Lemma, $\mathcal{C}(G)$ has at least one maximal element - but most likely many.

Theorem 1. Let $\theta$ be any congruence on a graph $G$. Then there is a 1-1 correspondence between the congruences $\alpha$ on $G$ satisfying $\theta \sqsubseteq \alpha$ and the congruences on $G / \theta$. Furthermore, the poset of all such congruences $\alpha$ is isomorphic to the poset of all the congruences on $G / \theta$.

Proof. Consider any graph $G=(V, E)$ with $\theta=\left(\sim_{\theta}, \widehat{E_{\theta}}\right)$ a (fixed) congruence on $G$ and let $\alpha$ be a congruence on $G$ with $\alpha=\left(\sim_{\alpha}, \widehat{E_{\alpha}}\right)$ and $\theta \sqsubseteq \alpha$, i.e., $\sim_{\theta} \subseteq \sim_{\alpha}$ and $\widehat{E_{\theta}} \subseteq \widehat{E_{\alpha}}-$ we shall abuse terminology by saying that $\alpha$ "contains" $\theta$.

Define a congruence $\alpha / \theta:=\left(\sim_{\alpha / \theta}, \widehat{E_{\alpha / \theta}}\right)$ on $G / \theta$ as follows: For all $v_{1}, v_{2} \in V$ we have

$$
\begin{array}{cll}
\left(\left[v_{1}\right]_{\sim_{\theta}},\left[v_{2}\right]_{\sim_{\theta}}\right) \in \sim_{\alpha / \theta} & \text { if and only if } & \left(v_{1}, v_{2}\right) \in \sim_{\alpha} \text { and } \\
{\left[v_{1}\right]_{\sim_{\theta}}\left[v_{2}\right]_{\sim_{\theta}} \in \widehat{E_{\alpha / \theta}}} & \text { if and only if } & v_{1} v_{2} \in \widehat{E_{\alpha}} .
\end{array}
$$


First we show that $\sim_{\alpha / \theta}$ and $\widehat{E_{\alpha / \theta}}$ are both well defined:

Assume $v_{1} \sim_{\theta} v_{1}^{\prime}$ and $v_{2} \sim_{\theta} v_{2}^{\prime}$. Since $\sim_{\theta} \subseteq \sim_{\alpha}$ it follows that $v_{1} \sim_{\alpha} v_{1}^{\prime}$ and $v_{2} \sim_{\alpha} v_{2}^{\prime}$. By the transitivity and symmetry of $\sim_{\alpha}$ it follows that $\left(v_{1}, v_{2}\right) \in \sim_{\alpha}$ if and only if $\left(v_{1}^{\prime}, v_{2}^{\prime}\right) \in \sim_{\alpha}$.

Starting again with $v_{1} \sim_{\theta} v_{1}^{\prime}$ and $v_{2} \sim_{\theta} v_{2}^{\prime}$ we have that $v_{1} v_{2} \in \widehat{E_{\alpha}}$ if and only if $v_{1}^{\prime} v_{2}^{\prime} \in \widehat{E_{\alpha}}$ by the substitution property of $\widehat{E_{\alpha}}$.

It is easy to see that $\sim_{\alpha}$ is an equivalence relation on $V(G / \theta)$.

In order to see that $E(G / \theta) \subseteq \widehat{E_{\alpha / \theta}}$, we note that $\left[v_{1}\right]_{\sim_{\theta}}\left[v_{2}\right]_{\sim_{\theta}} \in E(G / \theta)$ if and only if $v_{1} v_{2} \in \widehat{E_{\theta}}$, which implies that $v_{1} v_{2} \in \widehat{E_{\alpha}}$ and therefore $\left[v_{1}\right]_{\sim_{\theta}}\left[v_{2}\right]_{\sim_{\theta}} \in \widehat{E_{\alpha / \theta}}$.

The requirement that $\left(\left[v_{1}\right]_{\sim_{\theta}},\left[v_{2}\right]_{\sim_{\theta}}\right) \in \sim_{\alpha / \theta}$ implies $\left[v_{1}\right]_{\sim_{\theta}}\left[v_{2}\right]_{\sim_{\theta}} \notin \widehat{E_{\alpha / \theta}}$ follows immediately from the fact that $\alpha$ is a congruence, which means i.a. that $\left(v_{1}, v_{2}\right) \in \sim_{\alpha}$ implies that $v_{1} v_{2} \notin \widehat{E_{\alpha}}$.

To show that $\widehat{E_{\alpha / \theta}}$ satisfies the substitution property with respect to $\sim_{\alpha / \theta}$, let $\left[v_{1}\right]_{\sim_{\theta}}\left[v_{2}\right]_{\sim_{\theta}} \in \widehat{E_{\alpha / \theta}}$ and $\left[v_{1}\right]_{\sim_{\theta}} \sim_{\alpha / \theta}\left[v_{1}^{\prime}\right]_{\sim_{\theta}}$ and $\left[v_{2}\right]_{\sim_{\theta}} \sim_{\alpha / \theta}\left[v_{2}^{\prime}\right]_{\sim_{\theta}}$. Then the required conclusion follows from the substitution property of $\widehat{E_{\alpha}}$ with respect to $\sim_{\alpha}$, since by what is given we have that $v_{1} \sim_{\alpha} v_{1}^{\prime}$ and $v_{2} \sim_{\alpha} v_{2}^{\prime}$ so that $v_{1} v_{2} \in \widehat{E_{\alpha}}$ implies $v_{1}^{\prime} v_{2}^{\prime} \in \widehat{E_{\alpha}}$.

We conclude that $\alpha / \theta=\left(\sim_{\alpha / \theta}, \widehat{E_{\alpha / \theta}}\right)$ is indeed a congruence on $G / \theta$.

Next we consider the mapping $\bar{\pi}$ that maps every congruence $\alpha$ on $G$ that contains $\theta$ to the congruence $\alpha / \theta$ on $G / \theta$ described above. Let $\alpha$ and $\beta$ be any two congruences on $G$ which both contain $\theta$. First we show that this mapping satisfies $\sim_{\alpha} \subseteq \sim_{\beta}$ if and only if $\sim_{\alpha / \theta} \subseteq \sim_{\beta / \theta}$ :

The fact that $\left\{\left(v_{1}, v_{2}\right) \in \sim_{\alpha} \Longrightarrow\left(v_{1}, v_{2}\right) \in \sim_{\beta}\right\}$ if and only if $\left\{\left(\left[v_{1}\right]_{\sim_{\theta}},\left[v_{2}\right]_{\sim_{\theta}}\right) \in \sim_{\alpha / \theta} \Longrightarrow\left(\left[v_{1}\right]_{\sim_{\theta}},\left[v_{2}\right]_{\sim_{\theta}}\right) \in\right.$ $\left.\sim_{\beta / \theta}\right\}$ follows immediately from the definitions.

The fact that the mapping $\bar{\pi}$ also satisfies $\widehat{E_{\alpha}} \subseteq \widehat{E_{\beta}}$ if and only if $\widehat{E_{\alpha / \theta}} \subseteq \widehat{E_{\beta / \theta}}$ follows in a similar fashion immediately from the definitions.

By the above, $\bar{\pi}$ may be thought of as an order-embedding which is automatically (see p. 24 of [1]) an injection. To complete the proof, we need to show that $\bar{\pi}$ is a surjective mapping onto $\mathcal{C}(G / \theta)$. In order to do so, let $\gamma:=\left(\sim_{\gamma}, \widehat{E_{\gamma}}\right)$ be any congruence on $G / \theta$. In order to find a congruence $\alpha$ for which $\bar{\pi}(\alpha)=\gamma$, we first define a relation $\sim_{\alpha}$ on $G$ by

$$
v_{1} \sim_{\alpha} v_{2} \quad \text { if and only if }\left[v_{1}\right]_{\theta} \sim_{\gamma}\left[v_{2}\right]_{\theta} .
$$

Note that, if $v_{1} \sim_{\theta} v_{2}$, then $\left[v_{1}\right]_{\theta}=\left[v_{2}\right]_{\theta}$ and hence, by the reflexivity of $\sim_{\gamma}$, we have that $\left[v_{1}\right]_{\theta} \sim_{\gamma}\left[v_{2}\right]_{\theta}$ so that $v_{1} \sim_{\alpha} v_{2}$, i.e., $\sim_{\theta} \subseteq \sim_{\alpha}$. It also follows easily that $\sim_{\alpha}$ is an equivalence relation since $\sim_{\gamma}$ is one.

Next we define a set $\widehat{E_{\alpha}}$ (of unordered pairs of different elements of $V$ ) by

Firstly, $E \subseteq \widehat{E_{\alpha}}$ :

$$
v_{1} v_{2} \in \widehat{E_{\alpha}} \quad \text { if and only if }\left[v_{1}\right]_{\theta}\left[v_{2}\right]_{\theta} \in \widehat{E_{\gamma}} \text {. }
$$

Suppose $v_{1} v_{2} \in E$. Then $\left[v_{1}\right]_{\theta}\left[v_{2}\right]_{\theta} \in E(G / \theta) \subseteq \widehat{E_{\gamma}}$, i.e., $v_{1} v_{2} \in \widehat{E_{\alpha}}$.

Secondly, if $v_{1} \sim_{\alpha} v_{2}$, then $v_{1} v_{2} \notin \widehat{E_{\alpha}}$ :

Suppose $v_{1} \sim_{\alpha} v_{2}$. This means that $\left[v_{1}\right]_{\theta} \sim_{\gamma}\left[v_{2}\right]_{\theta}$ so that $\left[v_{1}\right]_{\theta}\left[v_{2}\right]_{\theta} \notin \widehat{E_{\gamma}}$ (since $\gamma$ is a congruence on $G / \theta$ ). But then $v_{1} v_{2} \notin \widehat{E_{\alpha}}$.

Thirdly, $\widehat{E_{\alpha}}$ satisfies the substitution property with respect to $\sim_{\alpha}$ :

If $v_{1} v_{2} \in \widehat{E_{\alpha}}, v_{1} \sim_{\alpha} v_{1}^{\prime}$ and $v_{2} \sim_{\alpha} v_{2}^{\prime}$, then $\left[v_{1}\right]_{\theta}\left[v_{2}\right]_{\theta} \in \widehat{E_{\gamma}},\left[v_{1}\right]_{\theta} \sim_{\gamma}\left[v_{1}^{\prime}\right]_{\theta}$ and $\left[v_{2}\right]_{\theta} \sim_{\gamma}\left[v_{2}^{\prime}\right]_{\theta}$. But then $\left[v_{1}^{\prime}\right]_{\theta}\left[v_{2}^{\prime}\right]_{\theta} \in \widehat{E_{\gamma}}$ (by the substitution property of $\widehat{E_{\gamma}}$ ), and hence $v_{1}^{\prime} v_{2}^{\prime} \in \widehat{E_{\alpha}}$.

From the above three claims we may conclude that $\alpha:=\left(\sim_{\alpha}, \widehat{E_{\alpha}}\right)$ is a congruence on $G$.

Let $\mathcal{C}(G, \theta):=\{\alpha \mid \alpha \in \mathcal{C}(G), \theta \sqsubseteq \alpha\}$. Using the above congruence $\alpha$ on $G$, defined from a given congruence $\gamma$ on $G / \theta$, we now can show that $\bar{\pi}: \mathcal{C}(G, \theta) \rightarrow \mathcal{C}(G / \theta)$ is surjective since $\bar{\pi}(\alpha)=\gamma$ : This is seen by merely remarking that, for a given congruence $\gamma$ on $G / \theta$ the conditions linking $\gamma$ to $\alpha$ and $\alpha$ to $\alpha / \theta$ are identical.

The isomorphism between the posets mentioned in the above theorem can be rephrased in terms of filters (also called dual ideals) in meet-semilattices (see [1]) as is now done in 
Corollary 1. Let $\theta$ be any congruence on a graph $G$. The filter $\mathcal{C}(G, \theta)$ of $\mathcal{C}(G)$ consisting of all the congruences $\alpha$ on $G$ satisfying $\theta \sqsubseteq \alpha$ and the meet-semilattice $\mathcal{C}(G / \theta)$ of all congruences on $G / \theta$ are isomorphic as complete meet-semilattices.

In the context of loopy graphs (Section 5) we shall return to these posets, which there become complete algebraic lattices.

\section{The isomorphism theorems}

We start with a theorem which might also be called (similar to the corresponding result for (universal) algebras in [3]) the Homomorphism Theorem for Graphs or (cf. [5]) the First Isomorphism Theorem for Graphs.

The kernel of a homomorphism: Given any graph homomorphism $f: G \longrightarrow H$, we define a congruence on $G$, denoted by $\theta_{f}$ and called the congruence induced by $f$ or the kernel of $f$, by

$$
\begin{aligned}
\theta_{f} & :=\left(\sim_{f}, \widehat{E_{f}}\right) \\
& :=\left(\left\{(x, y) \in V(G)^{2} \mid f(x)=f(y)\right\},\{u v \mid u, v \in V(G) \text { and } f(u) f(v) \in E(H)\}\right) \\
& =\left(f^{-1}\left[i d_{f(V(G))}\right], f^{-1}[E(H[f(V(G))])]\right) .
\end{aligned}
$$

We note that the equivalence classes of $\sim_{f}$ are the colour classes of the $H$-colouring $f$ of $G$. It is immediately clear that $\theta_{f}$ is a congruence on $G$.

Theorem 2. For any graph homomorphism $f: G \longrightarrow H$ we have that $G / \theta_{f}$ is isomorphic to $H[f(V(G))]$ under $[x] \mapsto f(x)$. When $f$ is surjective (i.e., $f(V(G))=V(H)$ ), then $G / \theta_{f}$ is isomorphic to $H$ under $[x] \mapsto f(x)$.

Proof. Note first that the mapping $[x] \mapsto f(x)$ is well defined since, if $[x]=[y]$, then $x \sim_{f} y$ and hence $f(x)=f(y)$ by the definition of $\theta_{f}$.

Furthermore we have that

$$
\begin{array}{rll}
{[x][y] \in E\left(G / \theta_{f}\right)} & \text { if and only if } \quad x y \in \widehat{E_{f}} \\
& \text { if and only if } \quad f(x) f(y) \in E(H) .
\end{array}
$$

Hence $G / \theta_{f}$ is isomorphic to $H[f(V(G))]$ under $[x] \mapsto f(x)$.

Theorem 2 demonstrates how the $f$-image $H[f(V(G))]$ outside $G$ in $H$ can be internalized within $G$ by the congruence $\theta_{f}$.

Corollary 2. Given $f: G \longrightarrow H$, we always have the commutative diagram

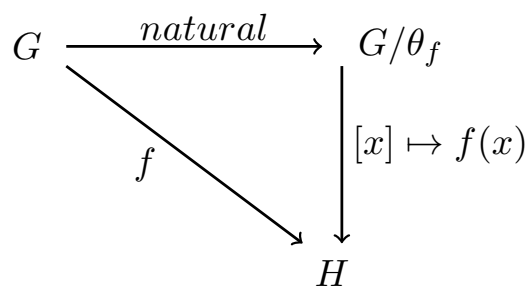

For purely algebraic structures (where every relation in the signature is a function, i.e., an operation on the underlying set of the structure) a congruence is simply an equivalence relation $\sim$ on the underlying set for which every relevant relation satisfies the substitution property with respect to $\sim$ (see [5] and [3]). For example, let $G$ be a group on the underlying set $U(G)$ and $I$ the binary relation which is the operation "inverse": $I=\left\{(x, y) \in U(G)^{2} \mid x y=y x=e\right\}$, and for $(x, y) \in I$ we write $y=x^{-1}$. The point is that for every $x$ there is exactly one $y$ such that $(x, y) \in I$. When then later defining $I$ in the naturally induced 
way on the elements (of the underlying set) of the quotient group $G / \sim$, there is exactly one element that $[x]^{-1}$ should be, namely $\left[x^{-1}\right](=[y]$ for $(x, y) \in I)$.

Now, for a graph $G=(V, E)$ the issue of defining "congruence on $G$ " aptly, is in a sense more complicated than for groups or algebraic structures in general. The simple reason is that when $x \in V$ there may be no $y$, or exactly one $y$, or more than one $y$ such that $x y \in E$. And similarly, given a homomorphism $f: G \rightarrow H, f(x)$ may have any number of neighbours in $H$, some of them within $f(V)$. This freedom has to be reflected in a freedom of choices for $\widehat{E}(\supseteq E)$ - which is not completely determined by $\sim$ and $E-$ in congruences $\theta=(\sim, \widehat{E})$ on $G$. Theorem 2 becomes impossible with only $\sim_{f}$ but without the freedom to stipulate $\widehat{E_{f}}$ as the "pullback" $f^{-1}[E(H[f(V)])]$ along $f$ of all and only those edges induced in the $f$-image inside $H$. As said, this insight originates in [4].

We now consider any graph $G=(V, E)$ and any congruence $\theta=(\sim, \widehat{E})$ on $G$, together with an induced subgraph $H \leq^{+} G$, the latter notation (read as " $H$ is an internal induced subgraph of $G$ ") implying that $H=\left(V_{H}, E_{H}\right)$ is an induced subgraph of $G$ with $V_{H} \subseteq V$. To define and explore interesting relationships between $\theta$ and $H$, we need some new concepts and their notations.

The congruence $\theta$ on $G$ induces the congruence $\theta_{H}=\left(\sim_{H}, \widehat{E_{H}}\right)$ on $H$, where

$$
\begin{aligned}
& \sim_{H}:=\sim \cap\left(V_{H} \times V_{H}\right) \text { and } \\
& \widehat{E_{H}}:=\left\{x y \in \widehat{E} \mid x, y \in V_{H}\right\} .
\end{aligned}
$$

That $\theta_{H}$ is indeed a proper congruence on $H$ is easy to verify. The congruence $\theta$ and $H$ induce a new internal induced subgraph of $G$, called the $\sim$-expansion of $H$ and denoted by $\sim(H) \leq^{+} G$, where

$$
\begin{aligned}
\sim(H) & :=\left(V_{\sim(H)}, E_{\sim(H)}\right), \\
V_{\sim(H)} & :=\left\{x \in V \mid(\exists y)\left(y \in V_{H}, x \sim y\right)\right\}, \text { and } \\
E_{\sim(H)} & :=\left\{x y \in E \mid x, y \in V_{\sim(H)}\right\} .
\end{aligned}
$$

Finally, just as $\theta$ induces the congruence $\theta_{H}$ on $H$, it induces a congruence $\theta_{\sim(H)}$ on $\sim(H)$ as follows: $\theta_{\sim(H)}:=\left(\sim \sim(H), \widehat{E_{\sim(H)}}\right)$, where

$$
\begin{aligned}
& \sim_{\sim(H)}:=\sim \cap\left(V_{\sim(H)} \times V_{\sim(H)}\right) \text { and } \\
& \widehat{E_{\sim(H)}}:=\quad\left\{x y \in \widehat{E} \mid x, y \in V_{\sim(H)}\right\} .
\end{aligned}
$$

We are now ready to formulate and prove a theorem which is related to the Second Isomorphism Theorem for algebraic structures (see [5], and [3], where it is called the First) and hence may be called the Second Isomorphism Theorem for Graphs.

Theorem 3. For any graph $G$, any congruence $\theta=(\sim, \widehat{E})$ on $G$ and any induced subgraph $H \leq^{+} G$, the following three graphs are pairwise isomorphic: $\sim(H) / \theta_{\sim(H)}, H / \theta_{H}$, and $(G / \theta)\left[\left\{[x]_{\sim} \mid x \in V_{H}\right\}\right]$, called $F$ in the figure below. .

Proof. We shall define three different surjective homomorphisms from the internal induced subgraph $\sim(H)$ of $G$ onto the three graphs of interest and call them $p: \sim(H) \rightarrow \sim(H) / \theta_{\sim(H)}, q: \sim(H) \rightarrow H / \theta_{H}$, and $r: \sim(H) \rightarrow(G / \theta)\left[\left\{[x]_{\sim} \mid x \in V_{H}\right\}\right]$. The kernels of these three homomorphisms will turn out to be the same: $\theta_{p}=\theta_{q}=\theta_{r}=\theta_{\sim(H)}$. By Theorem 2 each of the three graphs of interest is isomorphic to $\sim(H) / \theta_{\sim(H)}$, making them pairwise isomorphic.

Considering any $x \in V_{\sim(H)}$ we need to define $p(x) \in V\left(\sim(H) / \theta_{\sim(H)}\right)$. By the definition of $V_{\sim(H)}$ there exists some $x^{\prime} \in V_{H}$ with $x \sim x^{\prime}$. Define $p(x):=\left[x^{\prime}\right]_{\sim}=[x]_{\sim}$. Then $p$ is a homomorphism: if $x, y \in V_{\sim(H)}$ and $x y \in E_{\sim(H)}$, then $x y \in E$, and hence $x y \in \widehat{E}$, ensuring that $[x]_{\sim}[y]_{\sim} \in E\left(\sim(H) / \theta_{\sim(H)}\right)$. Picking any $[x]_{\sim} \in V\left(\sim(H) / \theta_{\sim(H)}\right)$, we have $[x]_{\sim}=\left[x^{\prime}\right]_{\sim}$ for some $x^{\prime} \in V_{H}$ and $x^{\prime} \in V_{\sim(H)}$ with $p\left(x^{\prime}\right)=[x]_{\sim}$, so $p$ is surjective. Obviously its kernel is $\theta_{\sim(H)}$ and $p$ is just the natural epimorphism.

Consider any $x \in V_{\sim(H)}$ with $x^{\prime} \in V_{H}$ and $x \sim x^{\prime}$. Define $q: V_{\sim(H)} \rightarrow V_{\left.H / \theta_{H}\right)}$ by $q(x)=\left[x^{\prime}\right]_{\sim_{H}}$. Then $q$ is a homomorphism: Consider $y \in V_{\sim(H)}$ with $x y \in E_{\sim(H)}$, hence $x y \in E$ and thus $x y \in \widehat{E}$. Therefore 

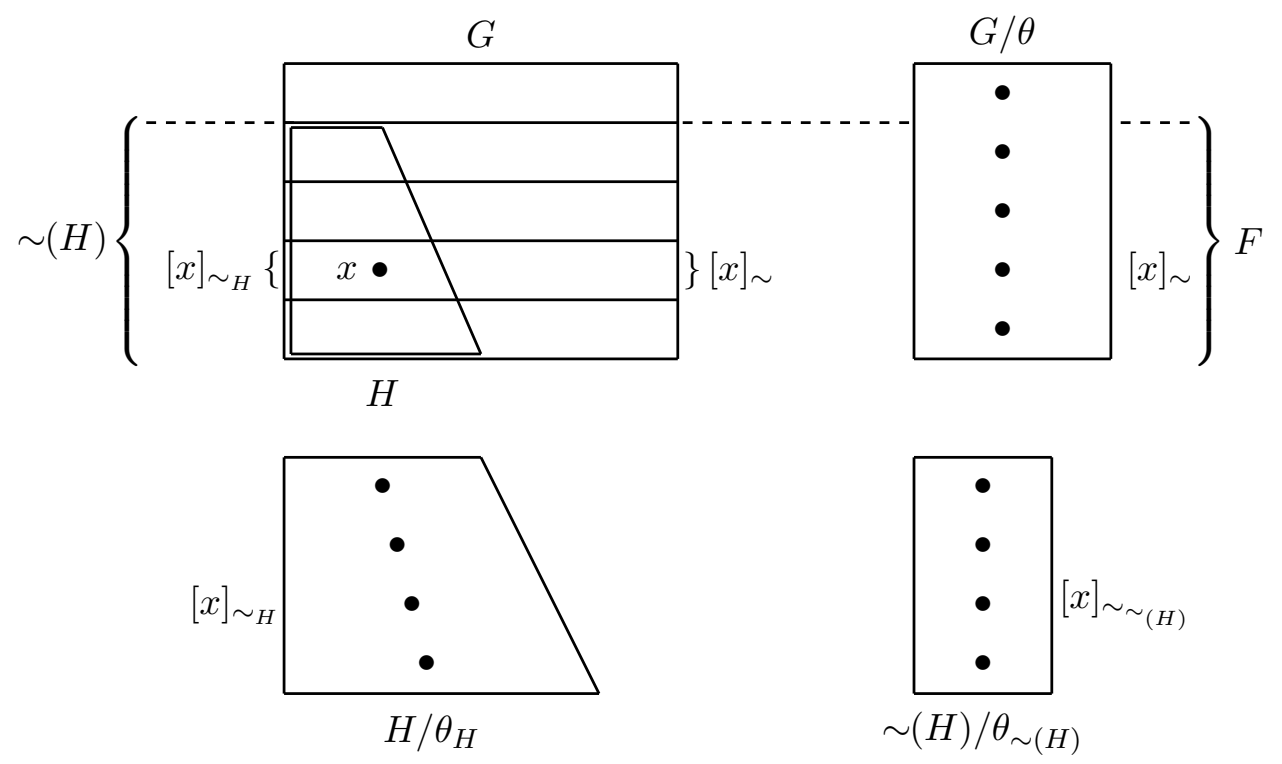

there are now $x^{\prime}, y^{\prime} \in V_{H}$ with $x \sim x^{\prime}, y \sim y^{\prime}$. By the substitution property of $\widehat{E}$ with respect to $\sim_{H}$ we have $x^{\prime} y^{\prime} \in \widehat{E}$, and hence $q(x) q(y)=\left[x^{\prime}\right]_{\sim_{H}}\left[y^{\prime}\right]_{\sim_{H}} \in E\left(H / \theta_{H}\right)$. Picking any $[x]_{\sim_{H}} \in V\left(H / \theta_{H}\right), x \in V_{H}$, so $x \in V_{\sim(H)}$ and $q(x)=[x]_{\sim_{H}}$, so $q$ is surjective. Its kernel is $\theta_{\sim(H)}$.

Finally, that $r: \sim(H) \rightarrow(G / \theta)\left[\left\{[x]_{\sim} \mid x \in V_{H}\right\}\right] ; x \mapsto[x]_{\sim}$ is a surjective homomorphism with kernel $\theta_{\sim(H)}$ needs no convoluted argument.

Next we develop an isomorphism theorem, called the Third Isomorphism Theorem for Graphs see [5] for a corresponding theorem in algebra, while in [3] it is called the Second-again as an application of the Homomorphism Theorem for Graphs.

Consider a graph $G=(V, E)$ and two of its congruences $\theta_{1}=\left(\sim_{1}, \widehat{E_{1}}\right)$ and $\theta_{2}=\left(\sim_{2}, \widehat{E_{2}}\right)$ with $\sim_{1} \subseteq \sim_{2}$ and $\widehat{E_{1}} \subseteq \widehat{E_{2}}$ - we have agreed to denote this by $\theta_{1} \sqsubseteq \theta_{2}$ in Section 2 . We define a congruence $\theta_{2 / 1}:=\left(\sim_{2 / 1}, E_{2 / 1}\right)$, which we also denote by $\theta_{2} / \theta_{1}$, of $G / \theta_{1}$ as follows: For all $x, y \in V$ we have

$$
\begin{aligned}
& \left([x]_{\sim_{1}},[y]_{\sim_{1}}\right) \in \sim_{2 / 1} \quad \text { if and only if } \quad(x, y) \in \sim_{2} \text { and } \\
& {[x]_{\sim_{1}}[y]_{\sim_{1}} \in \widehat{E_{2 / 1}} \quad \text { if and only if } \quad x y \in \widehat{E_{2}} .}
\end{aligned}
$$

In the above, $\widehat{E_{2 / 1}}$ can be considered as a set of unordered pairs (as it should) since $\widehat{E_{2}}$ is given as such. Note that the definition of $\theta_{2 / 1}$ above, where $\theta_{1}$ and $\theta_{2}$ are given congruences on $G$ with $\theta_{1} \sqsubseteq \theta_{2}$, imitates the definition in the proof of Theorem 1 of $\alpha / \theta$, where $\theta$ and $\alpha$ are given with $\theta \sqsubseteq \alpha$. The difference between this situation and that of Theorem 1 is that in the next result we shall compare two quotient graphs whereas in Theorem 1 we compare two posets. Because of this similarity (between $\alpha / \theta$ and $\theta_{2 / 1}$ ), we are already assured that each $\theta_{2 / 1}$ is a congruence on $G / \theta_{1}$.

Now we are ready to prove the Third Isomorphism Theorem for Graphs.

Theorem 4. For any graph $G=(V, E)$ and two congruences $\theta_{1}=\left(\sim_{1}, \widehat{E_{1}}\right)$ and $\theta_{2}=\left(\sim_{2}, \widehat{E_{2}}\right)$ of $G$ with $\theta_{1} \sqsubseteq \theta_{2}$ the quotient graph $\left(G / \theta_{1}\right) / \theta_{2 / 1}$ is isomorphic to $G / \theta_{2}$.

Proof. The proof is again an application of Theorem 2.

Consider the mapping $f:[x]_{\sim_{1}} \mapsto[x]_{\sim_{2}}$ from $G / \theta_{1}$ to $G / \theta_{2}$. This mapping is a homomorphism since, whenever $[x]_{\sim_{1}}[y]_{\sim_{1}} \in E\left(G / \theta_{1}\right)$, then $x y \in \widehat{E_{1}}$ and hence $x y \in \widehat{E_{2}}$ (since $\widehat{E_{1}} \subseteq \widehat{E_{2}}$ ), i.e., $[x]_{\sim_{2}}[y]_{\sim_{2}} \in$ $E\left(G / \theta_{2}\right)$. It is easy to see that $f$ is surjective. 
For the kernel $\left(\sim_{f}, \widehat{E_{f}}\right)$ of this homomorphism $f$ we see, from the definitions, that the pair $\left([x]_{\sim_{1}},[y]_{\sim_{1}}\right) \in$ $\sim_{f}$ if and only if $f\left([x]_{\sim_{1}}\right)=f\left([y]_{\sim_{1}}\right)$, i.e., $[x]_{\sim_{2}}=[y]_{\sim_{2}}$, which is equivalent to $(x, y) \in \sim_{2}$, while $[x]_{\sim_{1}}[y]_{\sim_{1}} \in \widehat{E_{f}}$ if and only if $f\left([x]_{\sim_{1}}\right) f\left([y]_{\sim_{1}}\right) \in E\left(G / \theta_{2}\right)$, i.e., $x y \in \widehat{E_{2}}$. These conditions, however, are exactly those in the definition of $\theta_{2 / 1}$ and therefore ensure that this kernel is $\theta_{2 / 1}$.

As remarked above, $\theta_{2 / 1}$ is a congruence of $G / \theta_{1}$ and by the above argument we have more than that: $\theta_{2 / 1}$ is indeed the kernel of the homomorphism $f$. The result now follows from Theorem 2 .

\section{Quotient graphs and subdirect products}

In this section we now link the above concepts in another way and prove a result which shows that the quotient of a graph with respect to the infimum of a given set of congruences on the graph is indeed a subdirect product of the quotient graphs of these congruences.

Theorem 5. Let $\theta_{i}, i \in I$, be congruences on a fixed graph $G=(V, E)$, while $\theta:=\bigwedge\left\{\theta_{i} \mid i \in I\right\}$. Then $G / \theta$ is isomorphic to a subdirect product of the quotient graphs $G / \theta_{i}$.

Proof. Using the notation as in the definitions in Section 1, we describe the required isomorphism, to be called $\alpha$, between $G / \theta$ and a suitable subdirect product of the $G_{i}:=G / \theta_{i}$ to prove the result: Let $\theta_{i}=\left(\sim_{i}, \widehat{E_{i}}\right)$ for each $i \in I$ and $\theta=\bigwedge\left\{\theta_{i} \mid i \in I\right\}=(\sim, \widehat{E})$. Note first that a typical vertex of $G / \theta$ is an equivalence class of $\bigcap\left\{\sim_{i} \mid i \in I\right\}$. Hence it is of the form $[x]$ with $y \in[x]$ iff $y \sim_{i} x$ for every $i \in I$. Furthermore, we should define $\alpha([x])$ to be an element of the vertex set of $\Pi_{i \in I} G_{i}$, i.e., a member of the Cartesian product $\Pi_{i \in I} V\left(G_{i}\right)$. Hence $\alpha([x])$ should be a function from $I$ to the disjoint union $\bigcup_{i \in I} V\left(G_{i}\right)$ (with prescribed properties).

This is achieved as follows: For any $x \in V$, we define $\alpha([x]):=f_{x}$, the function from $I$ to $\bigcup_{i \in I} V\left(G_{i}\right)$ which satisfies $f_{x}(i)=[x]_{\sim_{i}}$. We first note that $\alpha$ is well-defined, i.e., $\alpha([x])$ is not dependent on the choice of $x$ representing $[x]:$ If $[x]=[y]$, then $x \sim y$ so that $y \sim_{i} x$ for every $i \in I$ by the above, i.e., $[y]_{\sim_{i}}=[x]_{\sim_{i}}$ for every $i \in I$. Since these are the function values of $\alpha([y])$ and $\alpha([x])$, we can conclude that $\alpha$ is indeed well-defined. Note also that $[x]_{\sim_{i}}$ is a vertex of $G / \theta_{i}$ for every $i \in I$; thus it is immediately clear that this function satisfies the requirement to be a vertex of the Cartesian product $\Pi_{i \in I} G / \theta_{i}$.

Furthermore, the function $\alpha:[x] \mapsto f_{x}$ is an isomorphism since

1) $\alpha$ is an injection: If $[x] \neq[y]$, then $x \not y$ and hence $x \not_{i} y$ for some $i \in I$; for this $i$ we then have that $[x]_{\sim_{i}} \neq[y]_{\sim_{i}}$ so that $f_{x}(i) \neq f_{y}(i)$, i.e., $f_{x} \neq f_{y}$, and

2) $\alpha$ and $\alpha^{-1}$ are both edge preserving: for all $x, y \in V(G)$ we have

$$
\begin{array}{ll} 
& {[x][y] \in E(G / \theta)} \\
\text { if and only if } & x y \in \widehat{E} \\
\text { if and only if } & x y \in \widehat{E_{i}} \text { for all } i \in I \\
\text { if and only if } & {[x]_{\sim_{i}}[y]_{\sim_{i}} \in E\left(G / \theta_{i}\right) \text { for all } i \in I} \\
\text { if and only if } & f_{x} f_{y} \in E\left(\Pi_{i \in I} G / \theta_{i}\right) .
\end{array}
$$

We have now established that $\alpha(G / \theta)$ is an induced subgraph of $\prod_{i \in I} G / \theta_{i}$ and we need to prove that it is a subdirect product. Hence consider the $i$ th projection from $\Pi_{i \in I} G / \theta_{i}$ to $G / \theta_{i}$, i.e., the mapping $\alpha([x]) \mapsto \alpha([x])_{i}$. By the above, $\alpha([x])=f_{x}$ so that $\alpha([x])(i)=f_{x}(i)=[x]_{\sim_{i}}$ for all $i \in I$. In these equations, $x$ varies over all vertices from $G$; hence $[x]_{\sim_{i}}$ assumes all values from $V\left(G / \theta_{i}\right)$. So this projection is surjective.

If, in the above theorem, $\theta$ is the identity congruence on $G$, i.e., $\theta=\left(i d_{V(G)}, E(G)\right)$, then $G / \theta \cong G$. Hence we have

Corollary 3. If $\left\{\theta_{i} \mid i \in I\right\}$ is a set of congruences on a graph $G$ with $\bigwedge\left\{\theta_{i} \mid i \in I\right\}=\iota_{G}$, then $G$ is isomorphic to a subdirect product of the quotient graphs $G / \theta_{i}$. 
To illustrate the use of Corollary 3, we need the notion of a "universal vertex": A vertex in a graph is universal when it is adjacent to every other vertex of the graph. Note that every graph is the subdirect product of (some) graphs, in fact of one or more clones of itself: this can be seen by remarking that (e.g.) the mapping $v \mapsto(v, v)$ from $V(G)$ to $V(G \times G)$ for any graph $G$ maps the given graph onto the diagonal of the direct product in such a way that the projections are indeed surjective. In the next result we show that the "factor" graphs involved can almost always be restricted to graphs having universal vertices - this is certainly trivially the case if the graph $G$ itself has a universal vertex.

Theorem 6. Every graph except $2 K_{1}$, the graph with two vertices and no edge, is a subdirect product of graphs each of which has a universal vertex.

Proof. Let $G=(V, E)$ be any graph except $2 K_{1}$. If $G$ has only 1 or only 2 vertices, then $G$ has a universal vertex and the remark just before the theorem covers $G$. Hence we may assume that $|V| \geq 3$. Choose any vertex $v \in V$ and define the congruence $\theta_{v}:=\left(i d_{V}, \widehat{E_{v}}\right)$ on $G$, where $\widehat{E_{v}}:=E \cup\{v x \mid x \in V \backslash\{v\}\}$, ensuring that $v$ becomes adjacent in $\left(V, \widehat{E_{v}}\right)$, viewed as a graph, also to all vertices not previously in its neighbourhood. To show that $\bigwedge\left\{\theta_{v} \mid v \in V\right\}=\iota_{G}$, we need only prove that $E=\bigcap\left\{\widehat{E_{v}} \mid v \in V\right\}$. Clearly $E$ is contained in the intersection. Suppose edge $x y \in \widehat{E_{v}}$ for every $v \in V$, but $x y \notin E$. Since $|V| \geq 3$, there exists a $z \in V$ different from both $x$ and $y$. By the definition of $\widehat{E_{z}}$ we have $x y \notin \widehat{E_{z}}-$ a contradiction. So $\bigwedge\left\{\theta_{v} \mid v \in V\right\}=\iota_{G}$.

By Corollary 3, we now have that $G$ is isomorphic to a subdirect product of the (quotient) graphs $G / \theta_{v}$. It is easy to see that every $G / \theta_{v}$ has the universal vertex $\{v\}$.

It is also easy to see that $2 K_{1}$ is not a subdirect product of graphs each of which has a universal vertex since, if it were such a subdirect product, then each graph involved in the full direct product must have 2 vertices and hence be $K_{2}$. But then the direct product is $\Pi_{i \in I} K_{2}$, within which every subdirect product contains at least one induced $K_{2}$ - and hence cannot be $2 K_{1}$.

\section{Congruences on loopy graphs}

Lurking in the subconscious of graph theory there is a shoal involving ambiguity concerning the intuitive connotations of the existence (or not) of an edge or a path between two vertices. On the one hand one may perceive an edge as similar to a rigid bar of steel distancing the two vertices, forever keeping them apart. Then loops are proscribed: no vertex is distanced from itself, and no homomorphism can merge two adjacent vertices to a single one, as separation must be preserved. Simple graphs are loopless and instantiate this intuition.

Another intuition perceives edges and paths as links, realizing closeness and accessibility, keeping vertices together. Then loops at all vertices are prescribed: each vertex is already at itself, and homomorphisms can contract two adjacent vertices to a single one, preserving linkage. In this section only, the intuition of adjacency as togetherness prevails. All graphs here are loopy, meaning that there is an edge from every vertex to itself (while still eschewing multiple edges, directed edges, and labels). The graph $\bullet$ with nothing but one looped vertex is a homomorphic image of any graph. Congruences on loopy graphs are somewhat simpler than previously. Some new results can be reached under these alternate stipulations. No adjustment to the notion of homomorphism is needed for loopy graphs; it simply preserves adjacency.

An independent set (of vertices) of a loopy graph $G=(V, E)$ is a non-empty $W \subseteq V$ such that for all $x, y \in W$ if $x \neq y$, then $x y \notin E$.

A congruence on a loopy graph $G=(V, E)$ is a pair $\theta=(\sim, \widehat{E})$ such that

(i) $\sim$ is an equivalence relation on $V$ (hence $i d_{V} \subseteq \sim$ );

(ii $l$ ) $\widehat{E}$ is a set of unordered pairs of elements from $V$ with $E \subseteq \widehat{E}$ (hence $\{x x \mid x \in V\} \subseteq \widehat{E}$ );

(iiil) when $x \sim y$ then $x y \in \widehat{E}$ (i.e., $\sim$-equivalence classes induce complete subgraphs with respect to $\widehat{E}$ ); 
and

(iv) when $x, y, x^{\prime}, y^{\prime} \in V, x \sim x^{\prime}, y \sim y^{\prime}$, and $x y \in \widehat{E}$, then $x^{\prime} y^{\prime} \in \widehat{E}$.

Although we state ( $\mathrm{iii}_{l}$ ) explicitly to ease the comparison between the conditions for a congruence on a loopy graph to those in Section 1 for the case of simple graphs, $\left(\mathrm{iii}_{l}\right)$ - in the case of prescribed loops at every vertex - follows from (iv) : $x \sim y$ together with $x \sim x$ and the compulsory $x x \in \widehat{E}$, entail that $x y \in \widehat{E}$. That, in this section, every vertex is looped, requires no formal changes to the definition of the quotient of a graph modulo a congruence (in Section 1), nor to that of the kernel of a homomorphism (in Section 3) - though the semantics entailed by the implied congruence has shifted slightly.

The different intuitive connotations of the existence of an edge between two vertices (apartness in the case of simple graphs; togetherness in the case of loopy graphs) are expressed very explicitly in these different third conditions on a congruence: (iii) for simple graphs in Section 1 stipulates that vertices in the same -equivalence class - homomorphically mappable to a single image - should not be kept apart by any $\widehat{E}$-edge, while $\left(\mathrm{iii}_{l}\right)$ above for loopy graphs stipulates that the potential homomorphic collapse of an $\sim$-equivalence class to a singleton entails that every two vertices in such a class are already $\widehat{E}$-linked.

Using the same notation as in Section 2, we remark that for loopy graphs $G$ the congruence $\iota_{G}=$ $\left(i d_{V(G)}, E(G)\right)$ is the $\sqsubseteq$-minimum element of the partially ordered set $(\mathcal{C}(G)$; $\sqsubseteq)$ of all congruences on $G$. It should be clear that now, i.e., for a loopy graph $G, \tau_{G}:=(V(G) \times V(G),\{x y \mid x, y \in V(G)\})$ is the $\sqsubseteq$-largest element of $(\mathcal{C}(G) ; \sqsubseteq)$ and that $G / \tau_{G} \cong \bigodot$.

The fact that $(\mathcal{C}(G) ; \sqsubseteq)$ now has a largest element also enables us to describe, using the infima described in Section 2, the supremum of any number of congruences: For any set $\mathcal{D} \subseteq \mathcal{C}(G)$ we have

$$
\bigvee \mathcal{D}=\bigwedge\{\theta \mid \delta \sqsubseteq \theta \text { for every } \delta \in \mathcal{D}\}
$$

Following [1], we say that an element $k$ of a complete lattice $(L ; \leq)$ is compact if for every subset $S$ of $L$, if we have that $k \leq \bigvee S$, then $k \leq \bigvee F$ for some finite subset $F$ of $S$; the set of compact elements of $(L ; \leq)$ is denoted by $K(L)$. Furthermore, a complete lattice $(L ; \leq)$ is called algebraic if for every $a$ in $L$ we have that $a=\bigvee\{k \in K(L) \mid k \leq a\}$.

Theorem 7. The poset $(\mathcal{C}(G) ; \sqsubseteq)$ is a complete algebraic lattice for any loopy graph $G$.

Proof. $(\mathcal{C}(G) ; \sqsubseteq)$ is a complete lattice with respect to the meet operation described in Section 2 and the join operation described above. To show that it is indeed algebraic too, we remark that for every loopy graph $G=(V, E)$ and all vertices $a, b$ of $G$ with $a \neq b$, the pairs $\iota_{G},\left(i d_{V}, \widehat{E_{a b}}\right)$ and $\left(\sim_{a b}, \widehat{E_{a b}}\right)$ with $\sim_{a b}:=i d_{V} \cup\{(a, b),(b, a)\}$ and $\widehat{E_{a b}}:=E \cup\{a b\}$ are congruences on $G$. Moreover, these congruences are easily seen to be compact elements of the lattice $(\mathcal{C}(G)$; $\sqsubseteq$ ) and, finally, every congruence on the loopy graph $G$ is the join of all the compact elements of these three forms below it.

From Theorem 7 and (the analogue for loopy graphs of) Corollary 1 we now have

Corollary 4. Let $\theta$ be any congruence on a loopy graph $G$. The filter $\mathcal{C}(G, \theta)$ of $\mathcal{C}(G)$ and $\mathcal{C}(G / \theta)$ are isomorphic as complete algebraic lattices.

The atoms of the lattice $(\mathcal{C}(G) ; \sqsubseteq)$ : An atom (see for example [1]) in a lattice (or poset) $(L ; \leq)$ with least element 0 is an element $a$ which covers 0 , denoted by $0 \prec a$ and meaning that $0<a$ ( $\sqsubset$ in our notation) and there is no element of $L$ between 0 and $a$. The atoms of the lattice $(\mathcal{C}(G)$; $\sqsubseteq)$ are clearly the congruences of $G$ of the form $\left(i d_{V}, \widehat{E_{a b}}\right)$ with $\widehat{E_{a b}}:=E \cup\{a b\}$ (as in the proof) for any pair of vertices $a, b$ of $G$ with $a \neq b$ - since each such congruence is covered by the congruence $\left(\sim_{a b}, \widehat{E_{a b}}\right)$, we might refer to the latter congruences as near-atoms of the lattice. This lattice is therefore not only atomic (meaning, as in [1], that for every element $x \neq 0$ there is an atom $a$ with $a<x$ ); it is indeed near-atomistic, meaning that every non-zero element of the lattice is the join of the atoms and near-atoms below it.

We remark that, mutatis mutandis, Theorems 1 to 6 and Corollaries 1 to 3, reformulated for loopy graphs, still hold. A companion result of Theorem 6 in this context is 
Theorem 8. Any loopy graph with at least three vertices is a subdirect product of a set of graphs each of which is a clone of one of the four 3-vertex loopy graphs.

Proof. The four 3-vertex loopy graphs are $G_{0}, G_{1}, G_{2}$, and $G_{3}$.

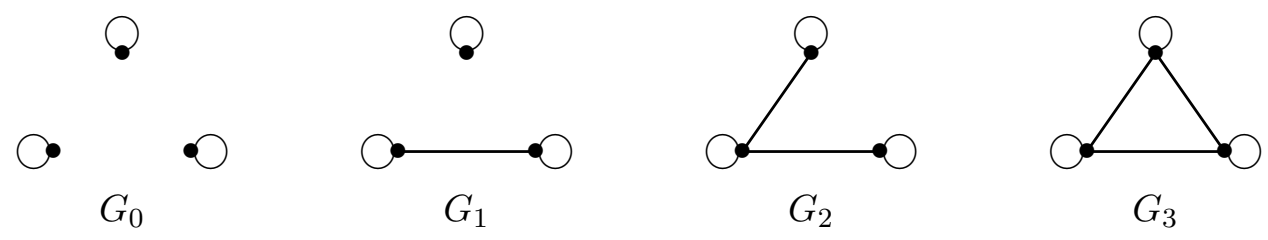

Let $G=(V, E)$ be a loopy graph with $|V| \geq 3$. A trichotomy of $V$ is a triplet $T=\left\{V_{1}, V_{2}, V_{3}\right\}$ of pairwise disjoint non-empty subsets of $V$ with $V_{1} \cup V_{2} \cup V_{3}=V$. We define $\mathcal{T}:=\{T \mid T$ is a trichotomy of $V\}$.

For every $T \in \mathcal{T}, T=\left\{V_{1}, V_{2}, V_{3}\right\}$, we define a congruence $\theta_{T}:=\left(\sim_{T}, \widehat{E_{T}}\right)$ on $G$ as follows:

$$
\begin{aligned}
\sim_{T}:= & \left(V_{1} \times V_{1}\right) \cup\left(V_{2} \times V_{2}\right) \cup\left(V_{3} \times V_{3}\right) \text { and } \\
\widehat{E_{T}}:=\left\{x y \mid(x, y) \in \sim_{T} \text { or }(\exists i, j \in\{1,2,3\})\left(\exists x^{\prime}, y^{\prime} \in V\right)\right. & \\
& {\left.\left[i \neq j ; x \sim_{T} x^{\prime} \in V_{i} ; y \sim_{T} y^{\prime} \in V_{j} ; \text { and } x^{\prime} y^{\prime} \in E\right]\right\} . }
\end{aligned}
$$

That $\theta_{T}$ is a congruence on $G$ is easy to verify. We claim that then $\bigwedge\left\{\theta_{T} \mid T \in \mathcal{T}\right\}=\iota_{G}$ and use, as can be expected, separate arguments to show that $i d_{V}=\bigcap\left\{\sim_{T} \mid T \in \mathcal{T}\right\}$ and that $E=\bigcap\left\{\widehat{E_{T}} \mid T \in \mathcal{T}\right\}$ :

Clearly, $i d_{V} \subseteq \bigcap\left\{\sim_{T} \mid T \in \mathcal{T}\right\}$, so we need only verify the reverse inclusion: If $x, y$ are distinct vertices of $G$, then we can create the trichotomy $T_{x, y}:=\{\{x\},\{y\}, V \backslash\{x, y\}\}$ of $V$, forcing $(x, y) \notin \sim_{T_{x, y}}$, so that $(x, y) \notin \bigcap\left\{\sim_{T} \mid T \in \mathcal{T}\right\}$. Hence $i d_{V}=\bigcap\left\{\sim_{T} \mid T \in \mathcal{T}\right\}$.

For the second equality, it is clear that $E \subseteq \bigcap\left\{\widehat{E_{T}} \mid T \in \mathcal{T}\right\}$ since, by the definition of $\widehat{E_{T}}, E \subseteq \widehat{E_{T}}$ for every $T$. (Note that when $x y \in E$, then either (a) $x$ and $y$ are in the same $V_{i}$, resulting in $(x, y) \in \sim_{T}$ and hence $x y \in \widehat{E_{T}}$; or else (b) $x \in V_{i}$ and $y \in V_{j}, i \neq j$, and hence again $x y \in \widehat{E_{T}}$.) We verify the reverse inclusion by showing that when $x y \notin E$, then $x y$ is not in the intersection. Suppose $x y \notin E$ (hence $x \neq y$ ) and consider the trichotomy $T_{x, y}=\{\{x\},\{y\}, V \backslash\{x, y\}\}$. Perusal of the definition of $\widehat{E_{T_{x, y}}}$ reveals that it does not have element $x y$.

Finally, it is easy to see that for each trichotomy $T$ the graph $G / \theta_{T}$ is a clone of one of the graphs $G_{0}, G_{1}, G_{2}$, or $G_{3}$. From Corollary 3 (now for loopy graphs) the theorem follows.

Corollary 5. Any complete loopy graph with at least three vertices is a subdirect product of clones of loopy $K_{3}$ (i.e., $G_{3}$ above). If the original graph has $n$ vertices $(n \geq 3)$, then the number of clones needed is the number of distinct trichotomies of an n-element set. (Note that the $k$ th direct power $G_{3}^{k}$ has $3^{k}$ vertices.)

\section{References}

[1] B. A. Davey and H. A. Priestly, Introduction to Lattices and Order, Second Edition, Cambridge University Press, New York, 2008.

[2] R. Diestel, Graph Theory, Fourth Edition, Graduate Texts in Mathematics 173, Springer, Heidelberg, 2010.

[3] G. Grätzer, Universal Algebra, Second Edition, Springer, Heidelberg, 2008.

[4] Susanna W. Malan, Relational Structures, Ph. D. thesis, Rand Afrikaans University, Johannesburg, 1976. 
[5] J. J. Rotman, An Introduction to the Theory of Groups, Fourth Edition, Graduate Texts in Mathematics 148, Springer, New York, 1995. 